\title{
Oral analgesia in fixed-time interval administration versus spinal morphine for post-Cesarean pain: a randomised controlled trial
}

\author{
Enav Yefet ${ }^{1,2,3}(1) \cdot$ Salih Nassar ${ }^{1} \cdot$ Julia Carmeli $^{4} \cdot$ Manal Massalha $^{1,6} \cdot$ Jamal Hasanein $^{5} \cdot$ Noah Zafran $^{1,6} \cdot$ \\ Michael Rudin ${ }^{4} \cdot$ Zohar Nachum $^{1,6}$
}

Received: 9 January 2021 / Accepted: 17 August 2021 / Published online: 31 August 2021

(c) The Author(s), under exclusive licence to Springer-Verlag GmbH Germany, part of Springer Nature 2021

\begin{abstract}
Purpose To compare the efficacy of fixed-time-interval oral analgesia and spinal-morphine for management of post-Cesarean pain.

Methods In this open-label, parallel-group, randomized, controlled trial, 200 women due to undergo elective Caesarean section with spinal anaesthesia were enrolled between July 2015 and April 2016. Patients were randomly assigned to receive either spinal fentanyl followed by oral doses of tramadol, paracetamol, and diclofenac at predetermined regular intervals of $6 \mathrm{~h}$ for the first $48 \mathrm{~h}$, and rescue treatment with percocet (oxycodone and paracetamol; oral analgesia group), or spinal morphine and rescue treatment with oral tramadol, paracetamol, and diclofenac (spinal-morphine group). The primary outcomes were pain intensity during the postoperative $48 \mathrm{~h}$, measured on a 10-point numeric rating scale (NRS) and expressed as area under the curve (AUC), and the number of breakthrough events of moderate to severe pain (defined as NRS score $\geq 4$ ).

Results The oral analgesia group compared to the spinal-morphine group had similar mean pain intensity (AUC $(120 \pm 35$ versus $121 \pm 31$, respectively; $p=0.8)$ but more events of moderate-to-severe breakthrough pain $(4.8 \pm 2$ versus $3.8 \pm 1.7$, respectively; $p=0.0002)$. Higher rates and longer durations of pruritus, nausea, and vomiting were reported among patients receiving spinal morphine, as compared with oral analgesia. Satisfaction scores were high in both groups ( $8.2 \pm 2.4$ versus $8.7 \pm 1.8$ in the oral analgesia and spinal morphine, respectively; $p=0.23$ ).

Conclusions Both oral analgesia at fixed time intervals and spinal morphine are satisfactory methods for treating postCaesarean pain.

Trial registration ClinicalTrials.gov Identifier: NCT02440399, date of registration: 07/05/2015. URL: https://clinicaltrials. gov/ct2/show/NCT02440399?term=enav+yefet\&rank=7.
\end{abstract}

Keywords Post-Caesarean pain $\cdot$ Spinal morphine $\cdot$ Oral analgesia $\cdot$ Postpartum

Enav Yefet

enavy1@gmail.com

1 Department of Obstetrics and Gynecology, Emek Medical Center, Afula, Israel

2 Department of Obstetrics and Gynecology, Baruch Padeh Medical Center Poriya, Tiberias, Israel

3 Azrieli Faculty of Medicine, Bar-Ilan University, Safed, Israel

4 Department of Anesthesiology, Emek Medical Center, Afula, Israel

5 Department of Neonatology, Emek Medical Center, Afula, Israel

6 Rappaport Faculty of Medicine, Technion, Haifa, Israel

\section{Introduction}

Post-Caesarean pain is an everyday challenge as Caesarean sections (CS) are one of the most prevalent laparotomies performed worldwide [1-3]. Inadequate maternal pain treatment has been suggested to impair maternal bonding, and decrease breastfeeding, infant care [4], and mobilisation, which may lead to thromboembolism [5, 6]. The importance of effective post-operative pain treatments is well acknowledged and a multimodal approach is taken by the obstetric team, anaesthesiologists and the nursing staff [7-11].

It is suggested that postoperative pain scores, measured using the visual analog scale (VAS), should be below 3, with patient satisfaction with their pain management $[12,13]$. Practice guidelines recommend the use of spinal opioids to 
treat post-Caesarean pain, because they have been shown to be more effective than intravenous opioids $[8,10]$. Yet, a second-line treatment in cases, where spinal morphine cannot be used, has not been established. Such cases include CS under general anesthesia, contraindications for spinal morphine, such as allergy, or history of severe adverse reactions after previous use, e.g., severe pruritus, gastrointestinal adverse effects, urinary retention, and respiratory depression [10]. While respiratory depression is rare in the obstetric population [14], continuous monitoring for 12-24 h postadministration is recommended $[15,16]$.

In our previous study, we have shown that oral analgesia based on paracetamol, diclofenac, and tramadol given at fixed time intervals, was effective in treating post-Cesarean pain, as demonstrated by a mean pain score below 3 in the first $48 \mathrm{~h}$ following surgery, a low adverse effect profile and high satisfaction rate [7]. Oral analgesia was shown to be effective in other studies as well [17, 18]. Its advantages include ease of administration, need for less cumbersome equipment, low cost and suitability after general anesthesia. However, its effectiveness and safety have not been sufficiently compared to that of spinal morphine. The present study compared the efficacy of fixed time-interval oral analgesia for post-Cesarean pain to that of the more traditional treatment with spinal morphine.

\section{Methods}

\section{Design}

An open-label, parallel-group, randomised, controlled trial was conducted at Emek Medical Center, a university-affiliated hospital in Afula, Israel. This study was authorised by the local review board of Emek Medical Center on March 24, 2015 (ID of the approval EMC-28-15), was performed in accordance with relevant guidelines and regulations, and was registered on http://www.clinicaltrials.gov (NCT02440399, principal investigator E.Y, date of registration: May 07, 2015).

Participants provided written informed consent. Women who intended to undergo elective Cesarean delivery with spinal anesthesia between July 2015 and April 2016, were recruited to this study. Eligibility and all baseline measures were taken during the preoperative evaluation and before randomization. Women were approached 1 day before the surgery or in the morning, when the CS was scheduled to the afternoon. Exclusion criteria were chronic pain, substance abuse, known allergy to any drug used in the study, obstructive sleep apnea, body mass index (BMI) $>40 \mathrm{~kg} / \mathrm{m}^{2}$, perioperative intravenous magnesium treatment, hypertension, renal failure, a history of severe post-operative nausea and vomiting and women who were designated to receive or who eventually underwent general anesthesia or who delivered vaginally.

Randomization of the groups was performed in a 1:1 ratio using a computer-generated randomization sequence; the randomization results were kept in sealed opaque envelopes, in a closed study box. The randomization sequence was concealed until intervention was assigned by physiciansinvestigators in this study.

\section{Interventions}

During recruitment, the study participants were instructed to report each time they suffered from pain or wanted additional analgesia regardless of the study group allocation. This instruction was repeated when they arrived to the maternity ward after surgery. In addition, women were asked to rank pain severity each time analgesia was administered or at least every $6 \mathrm{~h}$ by study nurses, who prior to the study had comprehensive guidance regarding the study protocol. Pain scores were recorded at rest for standardization. Pain assessment throughout the study was scored using the 10-point numeric rating scale (NRS), with whole integers (ranging from 0 (no pain) to 10 (worst pain)).

The same group of anesthesiologists performed all the spinal anesthesia procedures.

Following randomization, patients were randomly allocated to one of two groups.

1. Spinal morphine group-received spinal anesthesia with $150 \mu \mathrm{g}$ morphine and $10 \mathrm{mg}$ bupivacaine (isobaric) before surgery. Fentanyl was not given during the spinal anesthesia due to reports that morphine might contradict the short-acting effect of fentanyl when administered together [19]. Patients did not receive additional analgesia unless requested in the following manner: In the recovery ward, patients who complained of pain intensity of $\geq 5$ received intravenous $5 \mathrm{mg}$ morphine. Repeated doses were given in 10-min intervals if the patient still reported NRS score $\geq 5$. In the maternity ward, additional analgesia was given on demand or when reported NRS score for pain intensity was $\geq 4$ (rescue treatments). In such cases, patients received two tablets of Zaldiar (each tablet contained paracetamol $325 \mathrm{mg}$ and tramadol $37.5 \mathrm{mg}$ ) at minimal intervals of $6 \mathrm{~h}$, and $100 \mathrm{mg}$ diclofenac at minimal intervals of $12 \mathrm{~h}$ in the first $24 \mathrm{~h}$ from arrival to the maternity ward; an additional dose of diclofenac could be given after $48 \mathrm{~h}$ (Zaldiar and diclofenac were given simultaneously if the time frame allowed it).

2. Oral analgesia group-received spinal anesthesia with $25 \mu \mathrm{g}$ fentanyl and $10 \mathrm{mg}$ bupivacaine (isobaric) before surgery. Addition of fentanyl to intrathecal bupivacaine results in faster onset with improved peri-operative 
anesthesia, without increasing the side effects [20]. In the recovery ward, patients who suffered from pain received the same protocol of IV morphine described above. Before transfer to the maternity ward, all patients received a tablet of Percocet (oxycodone $5 \mathrm{mg}$ and paracetamol $325 \mathrm{mg}$ ). Once the patients arrived at the maternity ward, they received $100 \mathrm{mg}$ intravenous tramadol hydrochloride (the only time an IV medication was used), a tablet of $500 \mathrm{mg}$ paracetamol, and a tablet of $100 \mathrm{mg}$ diclofenac. Six hours after patient arrival and every $6 \mathrm{~h}$ thereafter, patients received two tablets of Zaldiar. The patients also received a tablet of $100 \mathrm{mg}$ diclofenac 12, 24, and $48 \mathrm{~h}$ after arrival. Patients were not woken for treatment. If additional analgesia was required, a tablet of percocet was given, as necessary, up to four times per day, at a minimal interval of 30 min (rescue treatment). The maximal allowed dose of paracetamol was $4 \mathrm{~g}$ per day. No woman in the study reached that dose. The oral analgesia protocol is the standard of care in our department.

In both study arms, pruritus was treated with promethazine. Nausea and vomiting were treated with granisetron and metoclopramide.

\section{Study outcomes}

The analysis focused on three main outcomes: (1) the analgesic effect of each treatment, (2) the safety profile and symptoms severity, and (3) patient satisfaction with the analgesia protocol. Two primary outcomes were addressed. The first was the sum of time-weighted NRS pain scores as an area under the curve (AUC) during the first $48 \mathrm{~h}$ post-CS for each participant. The curves were used to calculate the mean AUC for each cohort. An additional primary outcome was the number of events of breakthrough pain of moderate severity or higher, defined as NRS score $\geq 4$ during the first $48 \mathrm{~h}$ from patient arrival at the maternity ward. This outcome was selected since both analgesia treatment arms were designed to prevent breakthrough pain. An NRS score of 4 , which is considered moderate pain intensity, was chosen since it is the minimal score for which pain relievers can be offered by the nursing staff in our department. Breakthrough pain lasting up to $3 \mathrm{~h}$ was considered one event. After that, it was defined as a new event.

A structured questionnaire was used to evaluate the type, severity, and duration of adverse effects that may be related to the study medications. The events addressed included pruritus, nausea and vomiting, drowsiness, and an open question regarding any additional adverse effects, their duration and severity. Each adverse effect was scored on a scale ranging from 0 (not at all) to 10 (most severe). The questionnaires were distributed by investigators in this study (E.Y., Z.N.,
M.M., and S.N.) once from the third day post-surgery. Neonatal outcomes were also collected as described previously [7] and included altered consciousness, irritability, drowsiness, maximal neonatal bilirubin $(\mathrm{mg} / \mathrm{dL})$, need for phototherapy and adverse effects with possible relation to the pain medications or dehydration due to inadequate breastfeeding. The neonatal outcomes were documented by the nursing staff and physicians from the Department of Neonatology. The number of breastfeeds and supplemental formula used during the 5 days post-surgery (the standard hospitalization duration in our center) were also recorded.

Finally, patient satisfaction from each treatment protocol was evaluated using the questionnaire described above. Patients were asked if they would like the same treatment protocol in the future and whether they would recommend this treatment protocol to a friend. For each question, the patient gave a score from 1 (least satisfied/recommended) to 10 (most satisfied/recommended).

\section{Statistical analysis}

\section{Sample size}

As mentioned above, based on the available literature which demonstrated lower rates of breakthrough pain in the spinal morphine compared with oral analgesia [11], we hypothesised that spinal morphine will provide a superior analgesic effect.

In our previous study in which oral analgesia given at fixed time intervals was compared to oral analgesia administered on demand, the AUC of pain intensity during the 48-h post-surgery was around $140 \pm 50$ in the fixed time interval group [7]. Assuming a 20\% lower AUC for those receiving spinal morphine (i.e. $115 \pm 50$ ), the sample size required 85 women for each group (5\% 2 -sided alpha, $90 \%$ power).

In addition, in the same study, a maximum of four rescue treatments for breakthrough pain events were required. Based on these data, the sample size of 85 women for each arm is sufficient to detect a $25 \%$ reduction in the average number of breakthrough pain events from $4 \pm 2$ to $3 \pm 2$ in the oral analgesia group vs. the spinal morphine group, respectively (5\% 2-sided alpha, $90 \%$ power; $15 \%$ drop out was added). This sample size is sufficient to detect a mean difference of one event between the groups $( \pm 2$ standard deviations), which is the minimal possible difference.

Cohorts were compared at baseline using the Student $t$-test (or the Wilcoxon two sample test) for continuous variables and $\chi^{2}$ (or Fisher's exact test) for categorical variables. The number of women who suffered from moderate to severe breakthrough pain (NRS $\geq 4$ ) was evaluated in blocks of $6 \mathrm{~h}$ each, from patient arrival at the maternity ward to $48 \mathrm{~h}$ afterwards. The statistical analyses were performed using SAS 
9.4 software (SAS Institute Inc., Cary, NC, USA.). Statistical significance was determined if $p<0.05$.

The datasets generated and/or analysed during the current study are available from the corresponding author on reasonable request and subject to the directives of the institutional review board.

\section{Results}

Figure 1 describes the patient flow diagram. During the study period, 284 women underwent elective CS, including 12 who were not tested for eligibility since they

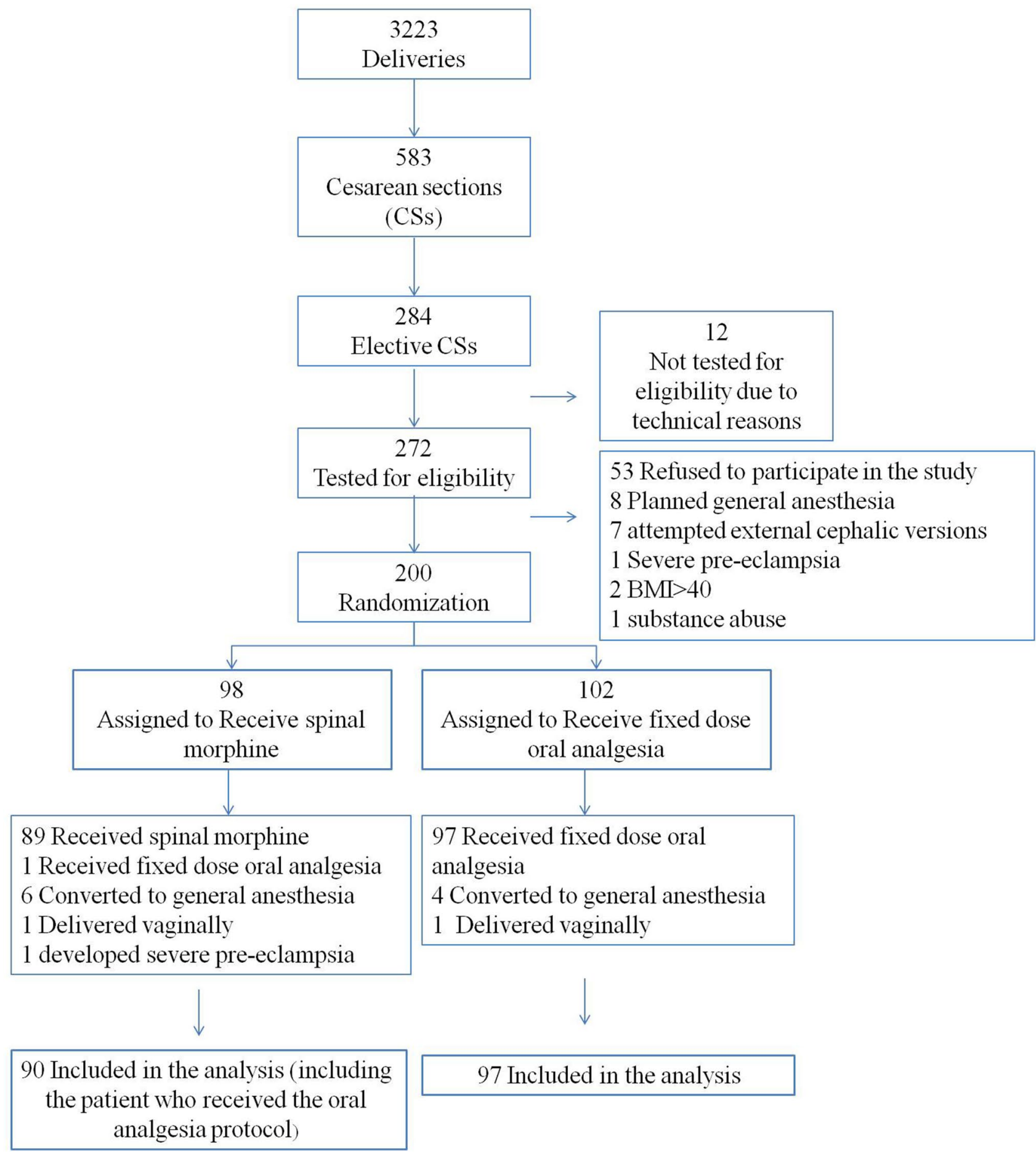

Fig. 1 Patient flow chart 
arrived on the weekend or were invited after cancelation of another patient. Of the 272 patients who were tested for eligibility, 253 women were eligible and 200 (80\%) were randomised. After randomisation, eight patients in the spinal morphine group and five patients in the oral analgesia group were excluded from the study since they met exclusion criteria (Fig. 1).

Of the remaining patients, 89 women were randomised to receive spinal morphine. One woman received the oral analgesia protocol since the anesthesiologist was concerned that the patient was allergic to morphine (which was ruled out post-surgery). Her data were analysed together with the spinal morphine group according to the intention to treat principle. The oral analgesia was administered to 97 women who were included in the analysis.

There were 94 and 104 newborns in the spinal morphine group and oral analgesia groups, respectively (including 4 and 7 pairs of twins, respectively). All women were interested in breastfeeding as the primary nutrition method.

The baseline characteristics of the mothers and neonates were not different between the groups (Table 1).

\section{Analgesia effect}

Maternal and neonatal outcomes are described in Table 2. The mean AUC for pain scores across the $48 \mathrm{~h}$ post-CS was similar between the cohorts $(120 \pm 35$ versus $121 \pm 31$ in the oral analgesia and spinal morphine cohorts, respectively, $p=0.8$ ). In contrast, women in the spinal morphine group had fewer events of moderate to severe breakthrough pain (NRS pain $\geq 4$ ) as compared with those receiving oral analgesia $(3.8 \pm 1.7$ versus $4.8 \pm 2.0$, respectively; $p=0.0002$ ). In addition, fewer women in the spinal morphine as compared to the oral analgesia group reported NRS $\geq 4$ for pain between 6 and $18 \mathrm{~h}$ post-arrival

Table 1 Demographic and obstetric characteristics of mothers and neonates

\begin{tabular}{|c|c|c|}
\hline Characteristic & Oral analgesia & Spinal morphine \\
\hline Maternal & $N=97$ & $N=90$ \\
\hline Maternal age (years) & $32.9 \pm 5.0,33(29-37)$ & $32.6 \pm 5.3,32(30-37)$ \\
\hline $\operatorname{BMI}\left(\mathrm{kg} / \mathrm{m}^{2}\right)$ & $25.6 \pm 5.0,24.5(21.7-29.2)$ & $25.8 \pm 5.5,24.6(21.8-28.4)$ \\
\hline Delivery week & $38.6 \pm 1.3,38.6(38.3-39.0)$ & $38.5 \pm 0.8,38.4(38.1-39.0)$ \\
\hline Preterm delivery ( $<37$ weeks) & $3(3 \%)$ & $2(2 \%)$ \\
\hline Parity & $2.9 \pm 1.4,3(2-3)$ & $2.7 \pm 1.0,3(2-3)$ \\
\hline Number of previous CSs & $1.2 \pm 1.0,1(0-2)$ & $1.2 \pm 1.0,1(0-2)$ \\
\hline Transverse corporal CS & $2(2 \%)$ & $0(0 \%)$ \\
\hline First CS & $29(30 \%)$ & $25(28 \%)$ \\
\hline \multicolumn{3}{|l|}{ Indications for $\mathrm{CS}$} \\
\hline Previous CS & $68(70 \%)$ & $66(73 \%)$ \\
\hline Malpresentation & $10(10 \%)$ & $13(14 \%)$ \\
\hline Multiple gestation & $6(6 \%)$ & $3(3 \%)$ \\
\hline Other & $13(13 \%)$ & $8(9 \%)$ \\
\hline Multiple spinal attempts & $4(4 \%)$ & $4(4 \%)$ \\
\hline Duration of CS (h) & $1.11 \pm 0.29,1.03[0.90-1.30]$ & $1.07 \pm 0.32,1.04[0.85-1.24]$ \\
\hline Morphine given at: PACU (mg) & $1.4 \pm 3.7,0(0-0)$ & $2.1 \pm 3.9,0(0-5)$ \\
\hline GDM & $7(7 \%)$ & $4(4 \%)$ \\
\hline Pre-GDM & $1(1 \%)$ & $2(2 \%)$ \\
\hline Neonate & $N=104$ & $N=94$ \\
\hline Twins & $14(13 \%)$ & $8(9 \%)$ \\
\hline Birth weight (g) & $3228 \pm 501,3215(2880-3548)$ & $3167 \pm 502,3123(2880-3465)$ \\
\hline Apgar score $1 \mathrm{~min}$ after delivery & $9.4 \pm 0.6,9(9-10)$ & $9.5 \pm 0.6,10(9-10)$ \\
\hline Apgar score 5 min after delivery & $9.98 \pm 0.1,10(10-10)$ & $9.98 \pm 0.2,10(10-10)$ \\
\hline Arterial cord $\mathrm{pH}$ & $7.30 \pm 0.05,7.30(7.28-7.33)$ & $7.29 \pm 0.05,7.29(7.26-7.31)$ \\
\hline Arterial cord $\mathrm{pH}<7.1$ & $1(1 \%)$ & $0(0 \%)$ \\
\hline Admission to the NICU & $4(4 \%)$ & $5(5 \%)$ \\
\hline
\end{tabular}

Values are given as mean \pm standard deviation, median (IQR) or number (percent)

$B M I$ body mass index, CS Cesarean section, GDM gestational diabetes mellitus, NICU neonatal intensive care unit, $P A C U$ post-anesthesia care unit 
Table 2 Maternal and neonatal outcomes

\begin{tabular}{|c|c|c|c|}
\hline $\begin{array}{l}\text { Outcome } \\
\text { Maternal }\end{array}$ & $\begin{array}{l}\text { Oral analgesia } \\
N=97\end{array}$ & $\begin{array}{l}\text { Spinal morphine } \\
N=90\end{array}$ & $p$ value \\
\hline Mean AUC for pain scores across first $48 \mathrm{~h}$ post-CS & $120 \pm 35,119(98-144)$ & $121 \pm 31,120(101-139)$ & 0.80 \\
\hline No. events per woman of moderate and severe pain (NRS pain $\geq 4)$ & $4.8 \pm 2.0,5(4-6)$ & $3.8 \pm 1.7,3(3-5)$ & 0.0002 \\
\hline No. events per woman of severe pain (NRS pain $\geq 6$ ) & $3.0 \pm 1.9,3(1-4)$ & $2.7 \pm 1.5,3(2-4)$ & 0.45 \\
\hline No. of times that analgesic agents were given during first $48 \mathrm{~h}$ & $5.4 \pm 1.8,5(4-7)$ & $3.3 \pm 1.8,3(3-5)$ & $<0.0001$ \\
\hline No. of times that analgesic agents were given beyond $48 \mathrm{~h}$ & $2.7 \pm 1.9,2(1-4)$ & $1.9 \pm 1.7,1(1-3)$ & 0.002 \\
\hline Satisfaction score from the analgesic treatment & $8.2 \pm 2.4,9(8-10)$ & $8.7 \pm 1.8,9(8-10)$ & 0.23 \\
\hline Requesting for the same analgesic protocol in the next CS score ${ }^{a}$ & $8.3 \pm 2.8,10(8-10)$ & $8.9 \pm 2.3,10(9-10)$ & 0.20 \\
\hline $\begin{array}{l}\text { Recommending the same analgesic treatment to a friend undergoing CS } \\
\text { score }^{\mathrm{a}}\end{array}$ & $8.4 \pm 2.8,10(8-10)$ & $8.9 \pm 2.3,10(9-10)$ & 0.26 \\
\hline Pruritus (severity score) ${ }^{* *}$ & $2.4 \pm 3.1,0(0-4)$ & $4.2 \pm 3.8,4(0-7)$ & 0.0007 \\
\hline Pruritus-severity score $\geq 5$ (number of patients) & $24(25 \%)$ & $43(48 \%)$ & 0.001 \\
\hline Pruritus (h) & $6.0 \pm 13.2,0(0-4)$ & $19.0 \pm 19.4,12(0-36]$ & $<0.0001$ \\
\hline Nausea (severity score) ${ }^{b}$ & $0.7 \pm 1.9,0(0-0)$ & $1.6 \pm 3.0,0(0-3)$ & 0.025 \\
\hline Nausea-severity score $\geq 5$ (number of patients) & $7(7 \%)$ & $15(17 \%)$ & 0.045 \\
\hline Nausea (h) & $1.4 \pm 5.6,0(0-0)$ & $4.1 \pm 10.9,0(0-1)$ & 0.04 \\
\hline Vomiting (h) & $0.3 \pm 1.5,0(0-0)$ & $3.7 \pm 9.4,0(0-0)$ & 0.001 \\
\hline Drowsiness (severity score) ${ }^{\mathrm{b}}$ & $1.3 \pm 2.8,0(0-0)$ & $1.1 \pm 2.6,0(0-0)$ & 0.40 \\
\hline Drowsiness (h) & $6.7 \pm 15.9,0(0-0)$ & $4.4 \pm 12.7,0(0-0)$ & 0.35 \\
\hline Adverse effects that bothered the patient (score) ${ }^{b}$ & $2.2 \pm 3.1,0(0-4)$ & $3.6 \pm 3.6,3(0-6)$ & 0.006 \\
\hline Adverse effects that bothered the patient-severity score $\geq 5$ & $24(25 \%)$ & $37(41 \%)$ & 0.02 \\
\hline $\begin{array}{l}\text { No. patients who reported that the severity of adverse effects justified treat- } \\
\text { ment avoidance }{ }^{b}\end{array}$ & $6(6 \%)$ & $11(12 \%)$ & 0.14 \\
\hline Total number of hospitalization days & $3.8 \pm 0.7,3.8(3.1-4.1)$ & $3.8 \pm 0.8,3.8(3.1-4.1)$ & 1 \\
\hline Neonate & $N=104$ & $N=94$ & $p$ value \\
\hline Maximal bilirubin $(\mathrm{mg} / \mathrm{dL})^{\mathrm{c}}$ & $8.0 \pm 2.9,8.5(6.4-10.2)$ & $8.2 \pm 3.0,8.7(6.5-10.1)$ & 0.65 \\
\hline Phototherapy (number of neonates) ${ }^{c}$ & $8(8 \%)$ & $4(4 \%)$ & 0.3 \\
\hline Jaundice (number of neonates) ${ }^{c}$ & $17(16 \%)$ & $18(19 \%)$ & 0.6 \\
\hline Breastfeeding (number of times) ${ }^{c}$ & $17.0 \pm 8.7,17(12-23)$ & $15.5 \pm 9.9,17(8-22)$ & 0.45 \\
\hline Use of supplemental formula (number of times) ${ }^{c}$ & $13.8 \pm 11.3,10(5-21)$ & $14.1 \pm 9.1,12.5(7-20)$ & 0.39 \\
\hline Change in neonatal weight at the time of discharge $(\mathrm{g})$ & $\begin{array}{l}-204 \pm 103,-201(-261 \\
\text { to }-155)\end{array}$ & $\begin{array}{l}-201 \pm 79,-205(-248 \\
\text { to } 155)\end{array}$ & 0.82 \\
\hline Respiratory distress & $3(3 \%)$ & $2(2 \%)$ & 1 \\
\hline
\end{tabular}

Values are given as mean \pm standard deviation, median (IQR) or number (percent). Satisfaction scores and adverse events profile were evaluated by structured questionnaires (one participant in the spinal morphine group did not answer the questionnaire)

$A U C$ area under the curve, $C S$ Caesarean section, NRS numeric rating scale

${ }^{\text {a }}$ These parameters were evaluated using a scale from 1 (not at all) to 10 (highly satisfied/recommended)

${ }^{\mathrm{b}}$ Severity score for adverse effect was evaluated using a scale from 0 (not at all) to 10 (most severe)

${ }^{\mathbf{c}}$ Refers to 5 days of hospitalisation

to the maternity ward (Fig. $2 ; p<0.05$ ). At the remaining times, pain scores were not statistically different between the groups (Fig. 2). There was no difference in the number of events of severe breakthrough pain $(\mathrm{NRS} \geq 6)$ between the spinal morphine and oral analgesia cohorts (Table 2).

\section{Adverse effect profile}

Data regarding the rate, severity, and duration of adverse effects related to the study medications are presented in Table 2. The spinal morphine group had a higher rate and 
Fig. 2 Breakthrough events of moderate to severe pain (NRS $\geq 4)$ in blocks of $6 \mathrm{~h}$ until $48 \mathrm{~h}$ after patient arrival to the maternity ward for women in the "spinal morphine" versus "oral analgesia" groups. The rate of women who reported NRS score for pain $\geq 4$ in each group is presented in blocks of $6 \mathrm{~h}$ for $48 \mathrm{~h} . * p=0.003$, $* * p=0.0001$

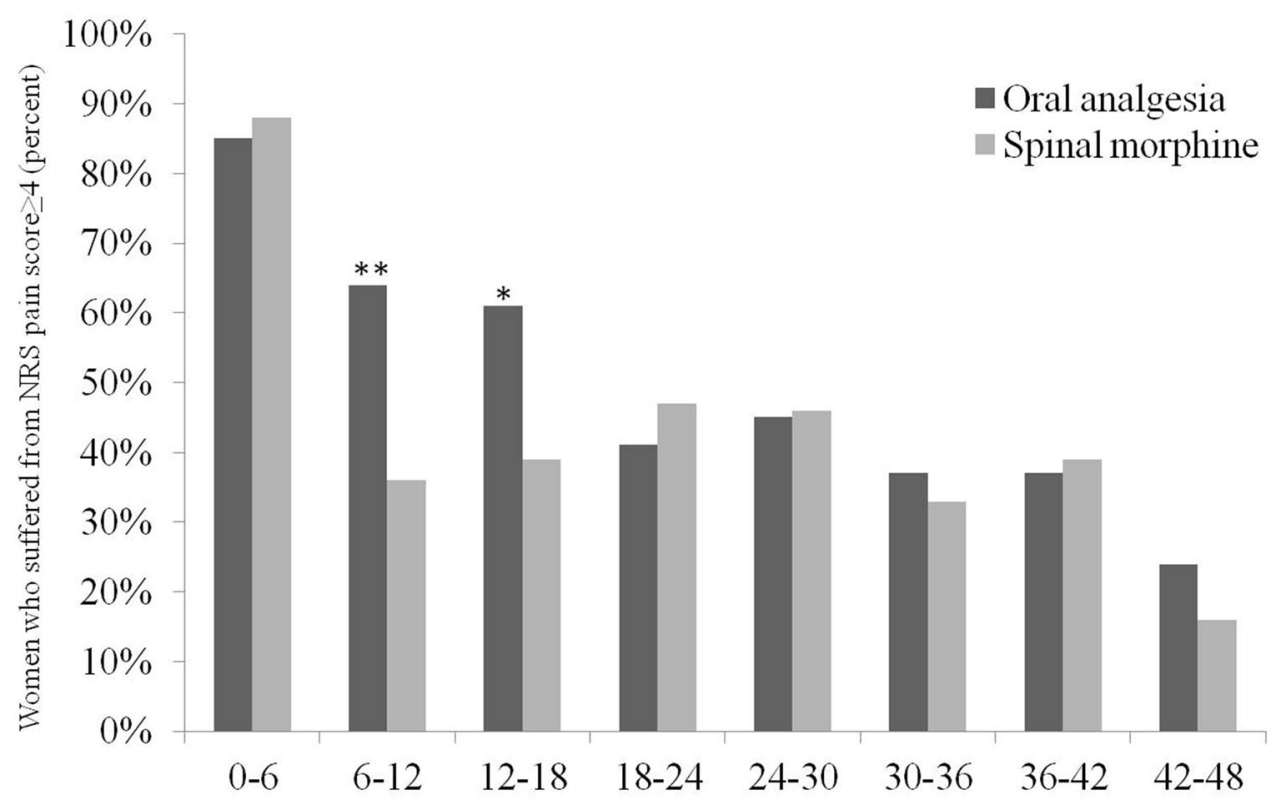

Hours from patient arrival to the department longer duration of pruritus, nausea, and vomiting compared with the oral analgesia group. Additionally, in each cohort, six women $(6 \%)$ reported dizziness $(p=0.9)$. In the spinal morphine group, one woman had a rash. In the oral analgesia group, two women reported headache, one woman reported leg weakness, one reported on sweats, and one had a case of burning sensation in the eyes.

The neonatal outcomes were comparable between the cohorts (Table 2). Five cases of respiratory distress were reported. In the spinal morphine group, one neonate, born in gestational week 38, needed non-invasive ventilator support. Another baby, born in gestational week 37, choked after feeding with supplemental formula. In the oral analgesia group, two babies had respiratory distress related to prematurity (gestational weeks 30 and 34) and one baby, born in gestational week 38, needed non-invasive oxygen support. Following meticulous investigation by the pediatricians, none of those events were considered related to the study medications.

\section{Patient satisfaction from the analgesia protocols}

Patient scores for satisfaction from the analgesia protocols, and willingness to undergo the same treatment protocol in the next surgery and to recommend it to a friend were high and not statistically different between the cohorts (Table 2).

\section{Discussion}

The present study compared the efficacy of oral analgesia administered at fixed time intervals for the treatment of post-Cesarean pain in the first $48 \mathrm{~h}$ following $\mathrm{CS}$ to that of spinal morphine. While the mean pain intensity was not different between the cohorts, spinal morphine was more effective in preventing breakthrough pain during the first 6-18 h, but was associated with a higher rate of adverse effects and satisfaction scores comparable with those for oral analgesia.

Both spinal opioids and oral analgesia protocols have been shown to be effective in treating post-operative pain [9, 10, 17, 18, 21-24]. However, a direct comparison of the two, in order to evaluate if oral analgesia could be an alternative for spinal morphine, has never been adequately studied. One study with a small sample size compared oral analgesia to spinal morphine. In the study, 120 women received either sustained-release oral oxycodone $20 \mathrm{mg}$ in the recovery room, followed by immediaterelease oxycodone $10 \mathrm{mg}$ 6-hourly for the first $24 \mathrm{~h}$, or intrathecal morphine $100 \mu \mathrm{g}$ at the time of spinal anesthesia. All women received postoperative paracetamol and 8-hourly diclofenac, starting at least $12 \mathrm{~h}$ postoperatively. 
Oxycodone and tramadol were used for rescue treatment. The AUC for pain scores across the first $24 \mathrm{~h}$ did not differ significantly between groups. Pruritus was reported less frequently in the oral analgesia group, but satisfaction scores were also lower in this group [11]. Higher intensity of worst pain in the 24 post-operative hours was suggested to contribute to these inferior satisfaction scores, underscoring the importance of addressing breakthrough pain as a primary endpoint. The study was limited by its small sample size, breakthrough pain being only a secondary outcome and the fact that oral analgesia was probably not given sufficiently, as suggested by the fact that $82 \%$ of the women in the oral analgesia group requested additional analgesia [11]. Breakthrough pain is a major challenge during postoperative care. In a previous study, around $68 \%$ women undergoing major gynecologic surgery had breakthrough pain requiring rescue analgesia within $48 \mathrm{~h}$ postoperatively, even though different continuous analgesia methods were given [25]. In the current study, the number of women with NRS $\geq 4$ between 24 and $48 \mathrm{~h}$ after arrival to the maternity ward was comparable between the cohorts, although the spinal morphine group did not receive any regular analgesia. This may be explained by the long-term effect of morphine, persisting longer than 24 hours, the low rate of breakthrough pain events in both cohorts for demonstrating any statistically significant difference, and that woman in the spinal morphine group received analgesia following demand that was sufficient to alleviate pain.

In our study, more women in the spinal morphine cohort reported pruritus that was difficult to manage. The exact mechanism of neuraxial opioid-induced pruritus is unclear [26] and its rate seems to be higher, affecting $>80 \%$ of postpartum patients even on administration of a low dose of $100 \mu \mathrm{g}$ intrathecal morphine [26]. Interaction of estrogen with opioid receptors was suggested to underlie the phenomenon [27, 28]. Our findings are also consistent with previous reports of shorter duration of pruritus following fentanyl treatment, as compared to pruritus invoked by spinal morphine $[11,26,29]$.

The oral analgesia protocol had a better safety profile than the spinal morphine protocol, but was less effective in providing pain relief, particularly during the $6-18 \mathrm{~h}$ following patient arrival at the maternity ward. The fact that women were not woken for analgesia administrations may have contributed to that. Multiple administrations at different times for each patient also complicated this protocol. A solution which was implemented in our department was fixed-interval dosing, administered when the patients were expected to be awake (e.g., at 6:00, 12:00, 18:00, and 24:00). Another way to simplify the protocol is to administer $400 \mathrm{mg}$ ibuprofen every $6 \mathrm{~h}$, together with Zaldiar instead of diclofenac. Future studies should examine whether a combination of spinal morphine and oral analgesia at fixed time intervals is superior to each method individually.

The strengths of this study were the high recruitment rate, its prospective randomised controlled design and the use of multiple endpoints for evaluating each treatment protocol. The limitations of this study included its single-center nature, the focus on short-term outcomes, and the open-label treatment administration, which is subject to bias. The types and nature of the questions in the structured questionnaire and the fact that this questionnaire was not validated may have also influenced the results, as previously suggested [30], yet, since both groups received the same questionnaire, this possible limitation was minimised. An additional limitation lay in the fact that the anaesthetic and post-analgesic regimens used in this study may not be in line with those used in other institutions. Nevertheless, the principle of using oral analgesia at fixed time intervals remains valid, regardless of the specific medications.

In conclusion, both spinal morphine and oral analgesia were shown to effectively prevent and alleviate post-CS pain, with high and comparable satisfaction scores. Spinal morphine was slightly better for pain prevention, however, at a cost of an increased adverse effect rate. Oral analgesia at fixed time intervals is a good alternative for spinal morphine in cases of general anaesthesia, contraindications for spinal morphine such as allergy, or substantial adverse effects.

Author contributions EY: project development, data collection, data analysis, manuscript writing. SN: project development, data collection, data analysis, manuscript editing. JC: project development, data collection, manuscript editing. MM: project development, data collection, manuscript editing. JH: project development, data collection, manuscript editing. NZ: project development, data collection, manuscript editing. MR: project development, data collection, manuscript editing. $\mathrm{ZN}$ : project development, data analysis, manuscript editing.

Funding This study was not funded by any organization.

\section{Declarations}

Conflict of interest All the authors declare that they have no conflict of interest.

Ethical approval All procedures performed in studies involving human participants were in accordance with the ethical standards of the Emek Medical Center institutional review board and with the 1964 Helsinki declaration and its later amendments.

Informed consent All patients gave a written informed consent form.

\section{References}

1. MacDorman MF, Menacker F, Declercq E (2008) Cesarean birth in the United States: epidemiology, trends, and outcomes. Clin Perinatol 35(2):293-307, v 
2. Sebastiao YV, Womack L, Vamos CA, Louis JM, Olaoye F, Caragan $T$ et al (2016) Hospital variation in Cesarean rates: contribution of individual and hospital factors in Florida. Am J Obstet Gynecol 214(1):123.e1-123.e18

3. Boyle A, Reddy UM (2012) Epidemiology of Cesarean delivery: the scope of the problem. Semin Perinatol 36(5):308-314

4. Karlstrom A, Engstrom-Olofsson R, Norbergh KG, Sjoling M, Hildingsson I (2007) Postoperative pain after Cesarean birth affects breastfeeding and infant care. J Obstet Gynecol Neonatal Nurs 36(5):430-440

5. Leung AY (2004) Postoperative pain management in obstetric anesthesia-new challenges and solutions. J Clin Anesth 16(1):57-65

6. Toglia MR, Nolan TE (1997) Venous thromboembolism during pregnancy: a current review of diagnosis and management. Obstet Gynecol Surv 52(1):60-72

7. Yefet E, Taha H, Salim R, Hasanein J, Carmeli Y, Schwartz N et al (2017) Fixed time interval compared with on-demand oral analgesia protocols for post-caesarean pain: a randomised controlled trial. BJOG 124(7):1067-1070

8. Practice Guidelines for Obstetric Anesthesia (2016) An updated report by the American Society of Anesthesiologists Task Force on Obstetric Anesthesia and the Society for Obstetric Anesthesia and Perinatology. Anesthesiology 124(2):270-300

9. Jakobi P, Weiner Z, Solt I, Alpert I, Itskovitz-Eldor J, Zimmer EZ (2000) Oral analgesia in the treatment of post-Cesarean pain. Eur J Obstet Gynecol Reprod Biol 93(1):61-64

10. Gadsden J, Hart S, Santos AC (2005) Post-Cesarean delivery analgesia. Anesth Analg 101(5 Suppl):S62-S69

11. McDonnell NJ, Paech MJ, Browning RM, Nathan EA (2010) A randomised comparison of regular oral oxycodone and intrathecal morphine for post-Caesarean analgesia. Int J Obstet Anesth 19(1):16-23

12. National Pharmaceutical Council, Inc (2001) Pain: current understanding of assessment, management, and treatments [monograph]. Joint Commission on Accreditation of Healthcare Organizations. https://www.npcnow.org/resources/pain-current-under standing-assessment-management-and-treatments

13. Pickering EH (2006) A pain relief after Caesarean section. In: Kinsella M (ed) Raising the standard: a compendium of audit recipes, 2nd edn. Royal College of Anaesthetists, London, pp 168-169

14. Abouleish E, Rawal N, Rashad MN (1991) The addition of $0.2 \mathrm{mg}$ subarachnoid morphine to hyperbaric bupivacaine for Cesarean delivery: a prospective study of 856 cases. Reg Anesth 16(3):137-140

15. Practice Guidelines for the Prevention (2016) Detection, and management of respiratory depression associated with neuraxial opioid administration: an updated report by the American Society of Anesthesiologists Task Force on Neuraxial Opioids and the American Society of Regional Anesthesia and Pain Medicine. Anesthesiology 124(3):535-552

16. Bauchat JR, Weiniger CF, Sultan P, Habib AS, Ando K, Kowalczyk JJ et al (2019) Society for Obstetric Anesthesia and Perinatology consensus statement: monitoring recommendations for prevention and detection of respiratory depression associated with administration of neuraxial morphine for Cesarean delivery analgesia. Anesth Analg 129(2):458-474

17. Zhong TD, Liu Q, Zhao JN, Wang HW, Konstantatos A (2014) A randomized trial to compare pain control using oral analgesia with epidural analgesia after Cesarean section following combined spinal-epidural anesthesia. Genet Mol Res 13(3):7086-7093

18. Lowder JL, Shackelford DP, Holbert D, Beste TM (2003) A randomized, controlled trial to compare ketorolac tromethamine versus placebo after Cesarean section to reduce pain and narcotic usage. Am J Obstet Gynecol 189(6):1559-1562

19. Weigl W, Bierylo A, Wielgus M, Krzemien-Wiczynska S, Kolacz M, Dabrowski MJ (2017) Perioperative analgesia after intrathecal fentanyl and morphine or morphine alone for Cesarean section: a randomized controlled study. Medicine (Baltim) 96(48):e8892

20. Bano F, Sabbar S, Zafar S, Rafeeq N, Iqbal MN, Haider S et al (2006) Intrathecal fentanyl as adjunct to hyperbaric bupivacaine in spinal anesthesia for caesarean section. J Coll Physicians Surg Pak 16(2):87-90

21. Dieterich M, Muller-Jordan K, Stubert J, Kundt G, Wagner K, Gerber B (2012) Pain management after Cesarean: a randomized controlled trial of oxycodone versus intravenous piritramide. Arch Gynecol Obstet 286(4):859-865

22. Davis KM, Esposito MA, Meyer BA (2006) Oral analgesia compared with intravenous patient-controlled analgesia for pain after Cesarean delivery: a randomized controlled trial. Am J Obstet Gynecol 194(4):967-971

23. Sammour RN, Ohel G, Cohen M, Gonen R (2011) Oral naproxen versus oral tramadol for analgesia after Cesarean delivery. Int $\mathrm{J}$ Gynaecol Obstet 113(2):144-147

24. Carvalho B, Mirza F, Flood P (2017) Patient choice compared with no choice of intrathecal morphine dose for caesarean analgesia: a randomized clinical trial. Br J Anaesth 118(5):762-771

25. Ismail S, Siddiqui AS, Rehman A (2018) Postoperative pain management practices and their effectiveness after major gynecological surgery: an observational study in a tertiary care hospital. J Anaesthesiol Clin Pharmacol 34(4):478-484

26. Kumar K, Singh SI (2013) Neuraxial opioid-induced pruritus: an update. J Anaesthesiol Clin Pharmacol 29(3):303-307

27. Krajnik M, Zylicz Z (2001) Understanding pruritus in systemic disease. J Pain Symptom Manag 21(2):151-168

28. LaBella FS, Kim RS, Templeton J (1978) Opiate receptor binding activity of 17-alpha estrogenic steroids. Life Sci 23(17-18):1797-1804

29. Reich A, Szepietowski JC (2010) Opioid-induced pruritus: an update. Clin Exp Dermatol 35(1):2-6

30. Chooi CS, White AM, Tan SG, Dowling K, Cyna AM (2013) Pain vs comfort scores after Caesarean section: a randomized trial. $\mathrm{Br}$ J Anaesth 110(5):780-787

Publisher's Note Springer Nature remains neutral with regard to jurisdictional claims in published maps and institutional affiliations. 